\title{
Application of Poisson distribution theory to the zona-free hamster oocyte penetration test to assess sperm function of men with asthenozoospermia
}

\author{
R. J. Aitken and R. A. Elton* \\ MRC Unit of Reproductive Biology, 37 Chalmers Street, Edinburgh EH3 9EW, and \\ ${ }^{*}$ Medical Computing and Statistics Unit, University of Edinburgh, Edinburgh EH8 9AG, U.K.
}

\begin{abstract}
Summary. Assessments of the penetrating potential of human spermatozoa were carried out using the zona-free hamster oocyte penetration test on 4 groups of subjects exhibiting (1) normal fertility, (2) idiopathic asthenozoospermia( $<40 \%$ motility), (3) asthenozoospermia associated with varicocoele and (4) oligoasthenozoospermia $\left(<20 \times 10^{6}\right.$ spermatozoa $/ \mathrm{ml}$ and $<40 \%$ motility). When the Poisson model was used to correct the results of the in-vitro penetration experiments for differences in motile sperm concentration, significant differences were apparent between the normal fertile controls and all 3 categories of asthenozoospermic patient. Furthermore, the penetrating ability of the motile spermatozoa from patients presenting with a varicocoele or oligoasthenozoospermia was significantly less than that for the group in which asthenozoospermia was the only detectable defect. These results emphasize the practical significance of the Poisson model in the analysis of male fertility and demonstrate that the asthenozoospermic condition is associated with a significant reduction in the fertilizing potential of the motile spermatozoa.
\end{abstract}

\section{Introduction}

Analysis of the functional competence of human spermatozoa has been facilitated by the introduction of the zona-free hamster oocyte penetration test by Yanagimachi, Yanagimachi \& Rogers (1976). This test assesses the ability of human spermatozoa to capacitate, undergo the acrosome reaction and generate a fusogenic equatorial segment capable of initiating fusion with the vitelline membrane of the oocyte (Yanagimachi, 1984: Aitken, Irvine, Clarkson \& Richardson, 1986). Although the test is not exhaustive, in that it does not examine the interaction between spermatozoa and the zona pellucida (Overstreet, Yanagimachi, Katz, Hayashi \& Hanson, 1980), its clinical significance has been demonstrated in retrospective and prospective studies (Rogers et al., 1979; Aitken et al., 1982a, b; Aitken, 1984; Aitken, Best, Warner \& Templeton, 1984).

We have described the significance of Poisson distribution theory in analysing the quantitative interaction between zona-free hamster oocytes and human spermatozoa (Aitken \& Elton, 1984). One of the consequences of this relationship was that equations could be constructed for correcting the outcome of the penetration test for differences in the concentration of motile spermatozoa in the incubation medium. It was envisaged that this facility would be particularly useful in cases of asthenozoospermia, for which the lack of motility limits the efficiency of layering techniques for isolating the motile spermatozoa.

Idiopathic asthenozoospermia ( $<40 \%$ motile spermatozoa) is present in about $10 \%$ of the infertile male population and is also a common attribute of the semen profile in cases of oligozoospermia and varicocoele, which together make up a further $60 \%$ of this group of patients (World Health Organisation, 1982). Whether asthenozoospermia is simply a matter of reduced sperm 
motility or is accompanied by defects in the fertilizing ability of the spermatozoa has implications for the treatment of this condition. In the present study we have addressed this subject by using the Poisson model to determine whether the penetrating potential of the motile spermatozoa in cases of asthenozoospermia differs significantly from that encountered in the normal fertile population.

\section{Materials and Methods}

\section{Patients}

Normal fertile donors who had fathered children $(\mathrm{N}=42)$ were recruited from a prevasectomy clinic; all had normal conventional semen profiles comprising $>20 \times 10^{6}$ spermatozoa $/ \mathrm{ml} ;>40 \%$ motility and $>40 \%$ normal morphology. Patients exhibiting idiopathic asthenozoospermia $(\mathrm{N}=24)$ were recruited from an infertility clinic if the semen profile was normal in terms of sperm density $\left(>20 \times 10^{6} / \mathrm{ml}\right)$ and morphology $(>40 \%$ normal) but $<40 \%$ motility had been recorded on at least two occasions within a 6-month period. Patients exhibiting oligoaesthenozoospermia $(\mathrm{N}=23)$ were recruited from the clinic if, on at least two occasions, the semen profile had been abnormal with respect to both sperm motility ( $<40 \%$ motile) and sperm number $\left(20<\times 10^{6} / \mathrm{ml}\right)$. Varicocoeles in 24 men were detected by palpation following the valsalva manoeuvre, while retrograde venous flux down the left spermatic vein was confirmed by examination with a Doppler stethoscope.

\section{Semen evaluation}

Semen samples were produced by masturbation, after at least $48 \mathrm{~h}$ abstinence, and collected in sterile plastic containers. After allowing at least $30 \mathrm{~min}$ for liquefaction to occur, a conventional semen analysis was carried out on each sample to determine sperm motility, morphology, total count and concentration, using the procedures laid down by the World Health Organisation (1980).

The ability of the spermatozoa to penetrate zona-free hamster oocytes was assessed at a fixed concentration of $10 \times 10^{6} \mathrm{cells} / \mathrm{ml}$ using the protocol described by Aitken, Wang, Liu, Best \& Richardson (1983c). The outcome of this procedure was described in terms of both the proportion of oocytes penetrated $(p)$ and the degree of polyspermy, i.e. the mean number of spermatozoa penetrating each oocyte $(m)$.

\section{Statistics}

Our previous study (Aitken \& Elton, 1984) suggested that the outcome of the zona-free hamster oocyte penetration test could be modelled using the Poisson distribution. For a semen sample exhibiting a motile sperm density of $x \times 10^{6} / \mathrm{ml}$ and an experiment involving $n$ oocytes, in which the mean number of spermatozoa penetrating each oocyte is $m$, the model is that $n m$, the total number of penetrating spermatozoa, has a Poisson distribution with expected value $n \theta x$. The parameter $\theta$ characterizes the in-vitro penetrating ability of the sperm sample and can be thought of as the mean number of spermatozoa penetrating each oocyte at a motile sperm concentration of $1 \times 10^{6} / \mathrm{ml}$. According to the model, the expected proportion of oocytes penetrated $(p)$ is related to the expected value of $m$ by the formula

$$
p=1-\mathrm{e}^{-\mathrm{m}}
$$

As a result of these relationships, the proportion of oocytes penetrated at any given theoretical concentration can be estimated using the formula

$$
p=1-\exp (-r m)
$$


where $r=$ the ratio of the theoretical concentration of motile spermatozoa to the observed concentration in the assay. In the present study this formula was used to correct each result obtained with the hamster oocyte assay to that which would have been observed at a fixed motile sperm concentration of $5 \times 10^{6} / \mathrm{ml}$. In this way the penetrating potential of the motile spermatozoa from normal fertile men could be directly compared with the results obtained with the asthenozoospermic patients, irrespective of any differences in the initial motility of the semen samples. The Mann-Whitney $U$ test was used to test whether differences could still be detected between groups after correcting the results for differences in motility.

One problem with this form of analysis arises when considering groups of patients exhibiting low motile sperm densities and a high frequency of zero penetration scores. In such instances it is possible that the absence of penetration is a consequence of an inadequacy in the number rather than the quality of gametes. For example an experiment with 20 oocytes and a $\theta$ value for the spermatozoa of 0.2 (typical for normal fertile men) would stand a $14 \%$ chance of giving a zero penetration score at a motile sperm density of $0.5 \times 10^{6} / \mathrm{ml}$ and a $45 \%$ chance of producing this result if the concentration of motile cells were reduced to $0.2 \times 10^{6} / \mathrm{ml}$. Since a sample giving zero penetration will retain its zero score even after correction with the above formulae, a group of samples exhibiting low concentrations of motile spermatozoa might appear less fertile than the fertile controls, even if the fertilizing potential of the motile spermatozoa $(\theta)$ was in the normal range. A method is therefore needed which takes the motile sperm concentration into account when assessing how likely it is that a zero penetration score indicates a low value of $\theta$. A statistical procedure for making these assessments has been devised using maximum likelihood methods (Fisher, 1963), the full details of which will be reported separately. In essence, the procedure recognizes that within any group of patients the value of $\theta$ varies from individual to individual and even between repeated samples from the same individual (Aitken \& Elton, 1984). We can therefore postulate that, within each group, $\theta$ varies from sample to sample according to a probability distribution over the range zero to infinity, and that differences in $m$ (corrected for motile sperm density) observed among the samples within each group, stem from this variability in $\theta$ as well as from the Poisson effect. We can then use maximum likelihood methods to estimate the parameters of the distribution of $\theta$ in each group and to test whether they are significantly different.

A suitable model for the variation of $\theta$ within groups is a Gamma distribution (Winer, 1971) which gives a variety of positively skewed shapes and has two parameters characterizing the mean value and standard deviation. These can be estimated by maximum likelihood methods and different groups can be compared using $\chi^{2}$ tests with two degrees of freedom to test for significant differences in these parameters.

\section{Results}

Table 1 summarizes the data on the degree of asthenozoospermia exhibited by the patients examined in this study and the outcome of the in-vitro penetration assays. As a consequence of the impaired motility observed in the original semen samples, the density of motile spermatozoa present in the incubation media was reduced from a mean of $5 \cdot 1 \times 10^{6} / \mathrm{ml}$ in the fertile controls to about $1.5 \times 10^{6}$ in the various groups of asthenozoospermic patients. The in-vitro penetration results were also much lower in these patients than in the normospermic controls, particularly in the groups characterized by oligoasthenozoospermia or the presence of varicocoeles, for whom the mean penetration rate fell below $10 \%$ (Table 1). In contrast, the normal fertile controls gave a mean penetration rate of $51 \cdot 8 \%$, while the patients exhibiting idiopathic asthenozoospermia produced an intermediate penetration result of $15.6 \%$. This gradation in penetrating potential, with the oligoasthenozoospermic samples exhibiting the poorest responses and the group exhibiting idiopathic asthenozoospermia occupying an intermediate position, was maintained when results were expressed in terms of the degree of polyspermy (mean number of spermatozoa penetrating 


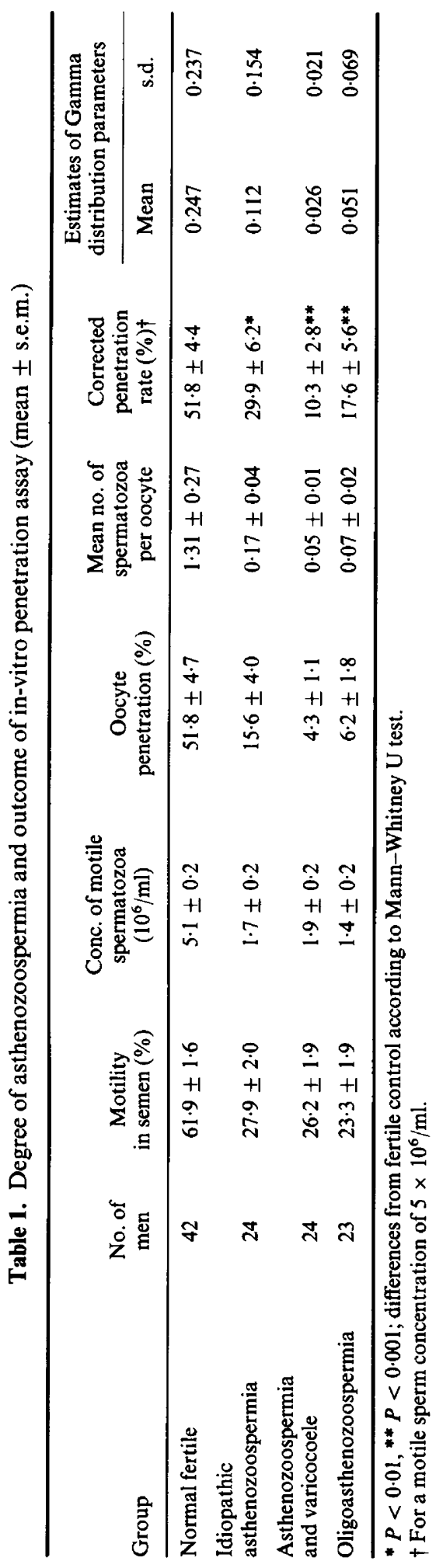




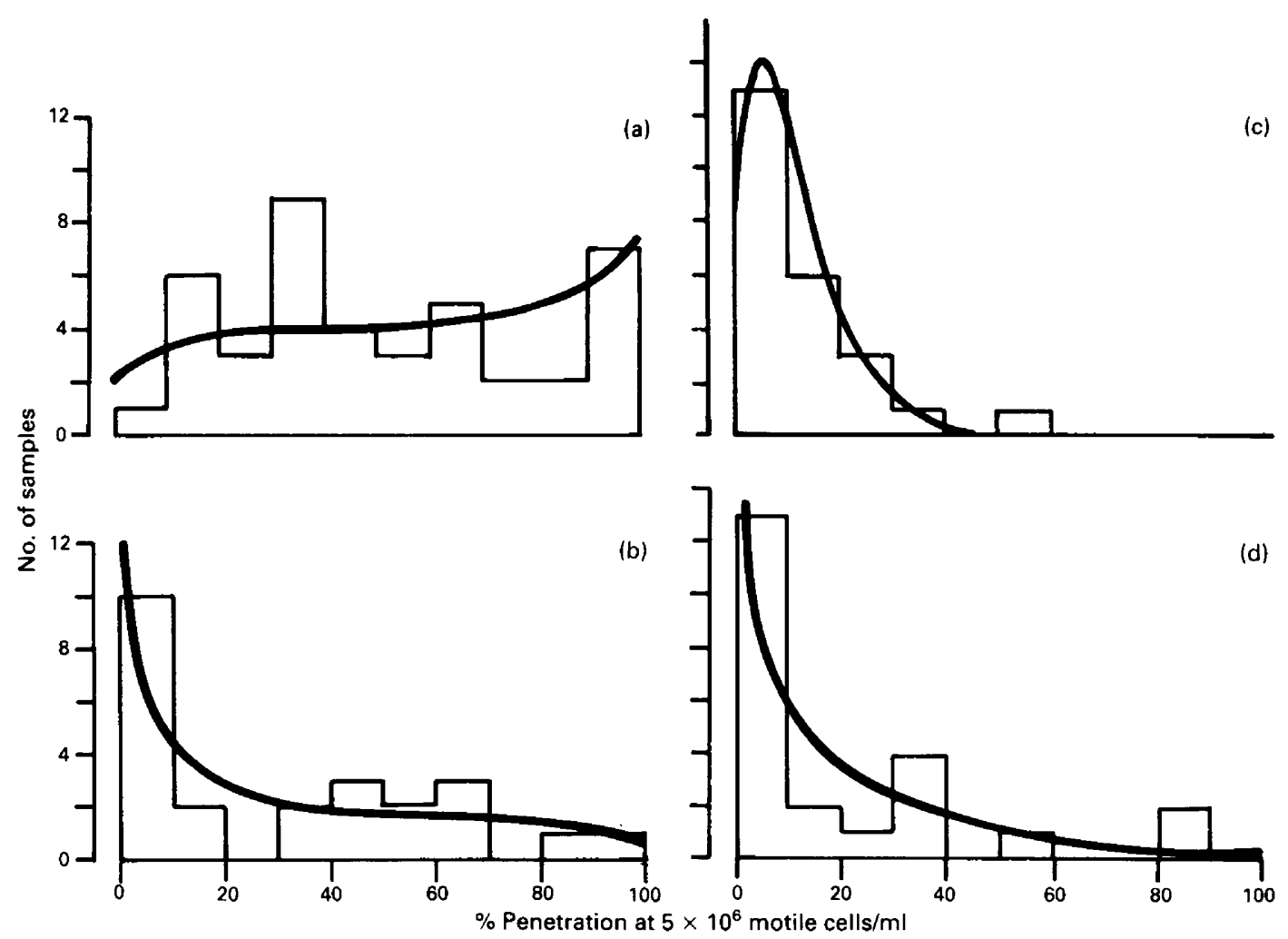

Fig. 1. Histogram: frequency distribution of sperm penetration rates with zona-free hamster oocytes, corrected for a motile sperm concentration of $5 \times 10^{6} / \mathrm{ml}$ for (a) normal fertile men, (b) men with idiopathic asthenozoospermia, (c) men with varicocoele and asthenozoospermia and (d) men with oligoasthenozoospermia. Continuous curves represent the fitted distributions of $\theta$ based on the Gamma theory.

each oocyte) and even after the results were corrected for a motile sperm concentration of $5 \times 10^{6} / \mathrm{ml}$ (Table 1). Frequency distributions for these corrected penetration rates are exhibited in Fig. 1. Also shown are the fitted distributions based on the Gamma theory, which appear to exhibit a satisfactory fit to the observed values.

When these corrected data were examined with the Mann-Whitney U test, significant differences were observed between the normal fertile group and the patients exhibiting idiopathic asthenozoospermia $(Z=2.70 ; P<0.01)$, asthenozoospermia in association with a varicocoele $(\mathrm{Z}=5.72 ; P<0.001)$ and oligoasthenozoospermia $(\mathrm{Z}=4.41 ; P<0.001)$. A significant difference in fertilizing potential was also apparent between the group exhibiting idiopathic asthenozoospermia and those patients presenting with varicocoeles $(Z=2.07 ; P<0.05)$, while a similar comparison between the idiopathic asthenozoospermic patients and those presenting with oligoasthenozoospermia showed no significance (Mann-Whitney $Z=1 \cdot 56$ ). Within the group of 24 varicocoele patients, 6 subjects presented semen samples on the day of analysis which were also oligozoospermic, in that the concentration of spermatozoa in the semen was $<20 \times 10^{6} / \mathrm{ml}$. To determine whether the poor fertilization results obtained with the varicocoele group were due to the inclusion of these oligozoospermic samples the analyses were repeated on the remaining 19 normospermic cases. This cohort of patients gave a mean \pm s.e. penetration rate of $11.3 \pm 3.6 \%$ at a motile sperm concentration of $5 \times 10^{6} / \mathrm{ml}$, which was still significantly lower than the values recorded for the normal fertile controls $(Z=4.99 ; P<0.001)$, although no longer quite 
significantly different from the group exhibiting idiopathic asthenozoospermia $(Z=1 \cdot 78$; $P<0 \cdot 10$ ). Similarly, no significant differences were apparent between the oligozoospermic and the varicocoele groups whether or not the latter included only the normospermic patients.

Analysis of the same data with the method of maximum likelihood in association with the Gamma distribution produced similar results, indicating that the high incidence of zero penetration results did not seriously affect the validity of the non-parametric analyses described above. When the in-vitro penetration rates were corrected for differences in motile sperm concentration, significant differences were apparent between the normal fertile population and the groups exhibiting idiopathic asthenozoospermia $\left(P<0.01 ; \chi^{2}=9.22 ; 2\right.$ d.f. $)$, varicocoele $\left(P<0.001 ; \chi^{2}=34.52\right.$; 2 d.f.) and oligoasthenozoospermia $\left(P<0.001 ; \chi^{2}=19.46 ; 2\right.$ d.f. $)$. The patients with idiopathic asthenozoospermia had significantly higher penetration rates than did those possessing varicocoeles $\left(P<0.01 ; \chi^{2}=10.64 ; 2\right.$ d.f. $)$ although the values for the oligoasthenozoospermic patients were not significantly different from those of patients with varicocoele $\left(\chi^{2}=2.46,2 \mathrm{~d}\right.$.f.) or idiopathic asthenozoospermia $\left(\chi^{2}=3 \cdot 15,2\right.$ d.f. $)$.

\section{Discussion}

This study has utilized the zona-free hamster oocyte penetration assay (Yanagimachi et al., 1976) to determine whether the asthenozoospermic condition is associated with a reduction in the fertilizing potential of the spermatozoa. Since this procedure does not examine certain aspects of sperm function, such as penetration of cervical mucus (Aitken, Sutton, Warner \& Richardson, 1985) or the competence of human spermatozoa to interact with the zona pellucida (Gould et al., 1983), false positive results are to be expected (Overstreet et al., 1980; Aitken, Ross \& Lees, 1983a). However, because the major property assessed by the penetration assay, sperm-oocyte fusion (Aitken et al., 1986), is a critical component of the fertilization process, poor or negative results should be of prognostic value (Aitken et al., 1984, 1986). The fact that the present study has revealed a high incidence of such low or negative results (Fig. 1) in association with asthenozoospermia suggests that this condition involves a loss of sperm function beyond a simple reduction in motility. The disappointing outcome of artificial insemination therapy in cases of asthenozoospermia is clearly in accord with this finding (Harris, Milligan, Masson \& Dennis, 1981).

Furthermore, analysis of the in-vitro penetration data using the Poisson model described by Aitken \& Elton (1984) has emphasized that the impaired penetration rates observed with these asthenozoospermic patients is not simply a consequence of the reduced numbers of motile spermatozoa because when the results were corrected to a constant concentration of motile cells, there were still differences between the asthenozoospermic patients and the normal fertile controls. Although no assessments of the quality of sperm movement were made in this study we have previously shown that the movement characteristics exhibited by human spermatozoa have little impact on the aspects of sperm function measured by the penetration test (Aitken et al., 1983a, 1985). The quality of sperm motility is clearly a major factor in determining the ability of human spermatozoa to penetrate the zona pellucida and cervical mucus, both of which are heavily dependent upon the shearing forces generated by sperm motility. In contrast, fusion between the vitelline membrane of the hamster oocyte and the human spermatozoon is dependent on gamete contact (Aitken et al., 1983a) rather than any specific attribute of sperm motility.

Statistical analysis of the corrected hamster oocyte penetration data revealed significant differences between the various groups of asthenozoospermic patients. In particular the presence of oligozoospermia or a varicocoele significantly reduced the degree of penetration achieved. While the poor fertilizing ability of oligoasthenozoospermic patients had been repeatedly demonstrated in artificial insemination studies (Dmowski, Gaynor, Lawrence, Rao \& Scommegna, 1979), the fact that the presence of a varicocoele significantly reduces the fertilizing potential of the 
asthenozoospermic patients is strong evidence that dilatation of the internal spermatic vein does make a significant contribution to the genesis of male infertility (Newton, Schinfeld \& Schiff, 1980; Vermeulen \& Vandeweghe, 1984). However, it has yet to be shown whether correction of the defect by embolisation of the spermatic vein will significantly influence the fertilising potential of these patients.

The mechanism by which asthenozoospermia is induced is not known although Hong, Chiang, Ku \& Wei (1984) have suggested that the spermatozoa of such patients may be unable to maintain a sufficiently low level of intracellular calcium. The inhibitory influence of free ionized calcium on ciliary and flagellar function is well known (Blum \& Hines, 1983) and it may be significant that the calcium chelating agents EGTA (ethylene glycol-bisB-aminoethyl ether $N, N, N^{\prime}, N^{\prime}$-tetra acetic acid) and EDTA (ethylene diamine tetra acetic acid) have a stimulatory effect on human sperm motility (Hong et al., 1984). The ability of the phosphodiesterase inhibitor, caffeine, to stimulate human sperm motility is apparently associated with the inhibition of calcium uptake (Peterson, Seyler, Bundman \& Freund, 1979). Calcium is also known to be essential for the processes of capacitation (Singh, Babcock \& Lardy, 1978), the acrosome reaction (Yanagimachi, 1982) and sperm oocyte fusion (Yanagimachi, 1978), all of which are assessed in the zona-free hamster oocyte system. It is therefore possible that the poor calcium homeostasis proposed for the spermatozoa of asthenozoospermic patients may also be responsible for the loss of fertilizing potential observed in the present study.

One possible effect of an imbalance in the levels of intracellular calcium may be the activity in the adenylate cyclase system. The fertilizing ability of human spermatozoa may be influenced by fluctuations in cAMP levels (Aitken, Best, Richardson, Schats \& Simm, 1983b) and the adenylate cyclase system of these cells is known to be inhibited by excess calcium, in the presence of the GTP analogue $5^{\prime}$-guanylylimido phosphate (Hyne \& Lopata, 1982). A second possibility is that high intracellular calcium levels may lead to the premature activation of phospholipase $A_{2}$, a calcium dependent enzyme which acts on membrane phospholipids to produce fusogenic lysophospholipids (Fleming \& Yanagimachi, 1981, 1984; Ohzu \& Yanagimachi, 1982; Llanos, Lui \& Meizel, 1982; Fleming \& Yanagimachi, 1984) as well as arachidonic acid. It is possible that one consequence of the premature, sustained activation of phospholipase activity by excessive intracellular calcium is the generation of reactive oxygen species (Smith \& Weidemann, 1980; Bromberg \& Pick, 1983; Lew, Wollheim, Waldvogel \& Pozzan, 1984) which might, in turn, lead to lipid peroxidation (Robak \& Sobanska, 1976) and a resultant loss of motility (Jones, Mann \& Sherins, 1978) as well as fertilizing potential.

We thank the staff of the Infertility Clinic, Edinburgh Royal Infirmary, and Jane Clarkson and David Richardson of the MRC Reproductive Biology Unit for help with this study.

\section{References}

Aitken, R.J. (1984) Diagnostic value of the hamster oocyte penetration assay. Int. J. Androl. 7, 273-275

Aitken, R.J. \& Elton, R.A. (1984) Significance of Poisson distribution theory in analysing the interaction between human spermatozoa and zona-free hamster oocytes. J. Reprod. Fert. 72, 311-321.

Aitken, R.J., Best, F.S.M., Richardson, D.W., Djahanbakhch, O., Mortimer, D., Templeton, A.A. \& Lees, M.M. (1982a) An analysis of sperm function in cases of unexplained infertility: conventional criteria, movement characteristics and fertilizing capacity. Fert. Steril. 38, 212-221.

Aitken, R.J., Best, F.S.M., Richardson, D.W., Djahanbakhch, O., Templeton, A.A. \& Lees, M.M. (1982b) An analysis of semen quality and sperm func- tion in cases of oligozoospermia. Fert. Steril. 38, 705-714.

Aitken, R.J., Ross, A. \& Lees, M.M. (1983a) Analysis of sperm function in Kartageners syndrome. Fert. Steril. 40, 696-698.

Aitken, R.J., Best, F., Richardson, D.W., Schats, R. \& Simm, G. (1983b) Influence of caffeine on movement characteristics, fertilizing capacity and ability to penetrate cervical mucus of human spermatozoa. J. Reprod. Fert. 67, 1927.

Aitken, R.J., Wang, Y-F., Liu, J., Best, F.S.M. \& Richardson, D.W. (1983c) The influence of medium composition, osmolarity and albumin content on the acrosome reaction and fertilizing capacity of human spermatozoa: development of an improved zona free 
hamster egg penetration test. Int. J. Androl. 6, 180-193.

Aitken, R.J., Best, F.S.M., Warner, P. \& Templeton, A. (1984) A prospective study of the relationship between semen quality and fertility in cases of unexplained infertility. J. Androl. 5, 297-303.

Aitken, R.J., Sutton, M., Warner, P. \& Richardson, D.W. (1985) Relationships between the movement characteristics of human spermatozoa and their ability to penetrate cervical mucus and zona-free hamster oocytes. J. Reprod. Fert. 73, $441-449$.

Aitken, R.J., Irvine, S., Clarkson, J.S. \& Richardson, D.W. (1986) Predictive value of in vitro sperm function tests. In Male Contraception and Future Prospects. Eds G. I. Zatuchni, A. Goldsmith, J. J. Sciarra \& J. Spieler. J. B. Lippincott, Philadelphia (in press).

Blum, J.J. \& Hines, M.A. (1983) Diffusion of $\mathrm{Ca}^{2+}$ and the quiescent response of sea urchin sperm flagella. $J$. theor. Biol. 100, 511-523.

Bromberg, Y. \& Pick, E. (1983) Unsaturated fatty acids as second messengers of superoxide generation by macrophages. Cell. Immunol. 79, 240-252.

Dmowski, W.P., Gaynor, L., Lawrence, M., Rao, R. \& Scommegna, A. (1979) Artificial insemination homologous with oligospermic semen separated on albumin columns. Fert. Steril. 31, 58-62.

Fisher, R.A. (1963) Statistical Methods for Research Workers. Oliver \& Boyd, Edinburgh.

Fleming, A.D. \& Yanagimachi, R. (1981) Effects of various lipids on the acrosome reaction and fertilizing capacity of guinea pig spermatozoa, with special reference to the possible involvement of hypophospholipid in the acrosome reaction. Gamete Res. 4, 253-293.

Fleming, A.D. \& Yanagimachi, R. (1984) Evidence suggesting the importance of fatty acids and fatty acid moieties of sperm membrane phospholipids in the acrosome reaction of guinea pig spermatozoa. $J$. exp. Zool. 229, 485-489.

Gould, J.E., Overstreet, J.W., Yanagimachi, H., Yanagimachi, R., Katz, D.F. \& Hanson, F.W. (1983) What functions of the sperm cell are measured by in vitro fertilization of zona-free hamster eggs? Fert. Steril. 40, $344-350$.

Harris, S.J., Milligan, M.P., Masson, G.M. \& Dennis, K.J. (1981) Improved separation of motile sperm in asthenospermia and its application to artificial insemination homologous (AIH). Fert. Steril. 36, $219-221$.

Hong, C.Y., Chiang, B.N., Ku, J. \& Wei, Y.H. (1984) Calcium chelators stimulate sperm motility in ejaculated human semen. Lancet i, 460-461.

Hyne, R.V. \& Lopata, A. (1982) Calcium and adenosine affect human sperm adenylate cyclase activity. Gamete Res. 6, 81-89.

Jones, R., Mann, T. \& Sherins, R.J. (1978) Adverse effects of peroxidized lipid on human spermatozoa. Proc. R. Soc. Lond. B 201, 413-417.

Lew, P.P., Wollheim, C.B., Waldvogel, F.A. \& Pozzan, T. (1984) Modulation of cytosolic-free calcium transients by changes in intracellular calcium-buffering capacity: correlation with exocytosis and $\mathrm{O}_{2}$ production in human neutrophils. J. Cell Biol. 99, 1212-1220.
Llanos, M.N., Lui, C.W. \& Meizel, S. (1982) Studies of phospholipase $\mathrm{A}_{2}$ related to the hamster sperm acrosome reaction. J.exp. Zool. 221, 107-117.

Newton, R., Schinfeld, J.S. \& Schiff, 1. (1980) The effect of varicocelectomy on sperm count, motility, and conception rate. Fert. Steril. 34, 250-254.

Ohzu, E. \& Yanagimachi, R. (1982) Acceleration of acrosome reaction in hamster spermatozoa by lysolecithin. J. exp. Zool. 224, 254-263.

Overstreet, J.W., Yanagimachi, R., Katz, D.F., Hayashi, K. \& Hanson, F.W. (1980) Penetration of human spermatozoa into the human zona pellucida and the zona-free hamster egg: a study of fertile donors and infertile patients. Fert. Steril. 33, 534-542.

Peterson, R.N., Seyler, D., Bundman, D. \& Freund, M. (1979) The effect of theophylline and dibutyryl cyclic AMP on the uptake of radioactive calcium and phosphate ions by boar and human spermatozoa. $J$. Reprod. Fert. 55, 385-390.

Robak, J. \& Sobanska, B. (1976) Relationship between lipid peroxidation and prostaglandin generation in rabbit tissues. Biochem. Pharmacol. 25, 2233-2236.

Rogers, B.J., van Campen, H., Ueno, M., Lambert, H., Bronson, R. \& Hale, R. (1979) Analysis of human spermatozoal fertilizing ability using zona-free ova. Fert. Steril. 32, 664-670.

Singh, J.P., Babcock, D.F. \& Lardy, H.A. (1978) Increased calcium-ion influx is a component of capacitation of spermatozoa. Biochem. J. 172, 549-556.

Smith, R.L. \& Weidemann, M.J. (1980) Reactive oxygen production associated with arachidonic acid metabolism by peritoneal macrophages. Biochem. Biophys. Res. Commun. 97, 973-980.

Vermeulen, A. \& Vendeweghe, M. (1984) Improved fertility after varicocele correction: fact or fiction? Fert. Steril. 42, 249-256.

Winer, B.J. (1971) Statistical Principles in Experimental Design, 2nd edn. McGraw-Hill, New York.

World Health Organisation (1980) Laboratory Manual for the Examination of Human Semen and SemenCervical Mucus Interaction. Eds M. A. Belsey, R. Eliasson, A. J. Galegos, K. S Moghissi, C. A. Paulsen \& M. R. N. Prasad. Press Concern, Singapore.

World Health Organisation (1982) Eleventh Annual Report. WHO Special Programme of Research Development and Research Training in Human Reproduction. World Health Organisation, Geneva.

Yanagimachi, R. (1978) Calcium requirement for spermegg fusion in mammals. Biol. Reprod. 19, 949-958.

Yanagimachi, R. (1982) Requirement of extracellular calcium ions for various stages of fertilization and fertilization-related phenomena in the hamster. Gamete Res. 5, 323-344.

Yanagimachi, R. (1984) Zona-free hamster eggs: their use in assessing fertilizing capacity and examining chromosomes of human spermatozoa. Gamete Res. 10, 187-232.

Yanagimachi, R., Yanagimachi, H. \& Rogers, B.J. (1976) The use of zona free animal ova as a test system for the assessment of the fertilizing capacity of human spermatozoa. Biol. Reprod. 15, 471-476. 